plementation of a new protocol by the Kansas City, Missouri Emergency Medical Services System. A total of 492 primary cardiac arrests were analyzed in this study.

The new protocol differed in several areas from the previous protocol modeled on the American Heart Association 2000 cardiac arrest guidelines. Chest compressions increased from a 5:1 ratio to a 50:2 ratio and were a mandatory initial action, before defibrillation, in any arrest that was not reliably witnessed within 5 minutes. A manual defibrillator was used if the paramedics identified a shockable rhythm after 200 compressions. Only after 3 cycles of 200 compressions was intubation attempted.

Outcome measures included survival to discharge and return of spontaneous circulation, both of which improved with the new protocol. Survival to discharge increased from $22 \%$ to $44 \%$. Return of spontaneous circulation improved with the new protocol and was most noticeable in the group of patients with witnessed ventricular fibrillation rising from $38 \%$ to $60 \%$. This study showed a benefit of this alternate approach to pre-hospital arrest.

(Circulation. 2009;119:2597-2605) AG Garza, MC Gratton, JA Salomone, et al.

Prepared by Anil Menon, MD, Stanford Wilderness Fellow, Palo Alto, CA, USA

\section{JOURNAL OF EMERGENCY MEDICINE}

\section{In-Flight Thoracic Ultrasound Detection of Pneumothorax in Combat}

This case report from the conflict in Afghanistan describes the use of ultrasonography to diagnose and change the management of a decompensating patient during medical evacuation via helicopter. The Afghani blast victim detailed in this case suffered shrapnel injuries to his head, neck, and lower extremities without obvious chest trauma. Initially, he was intubated for a low Glascow Coma Scale, ventilated at a rate of 10 breaths per minute on $100 \%$ oxygen, with vital signs as follows: heart rate 110 beats per minute, blood pressure 138/76 $\mathrm{mm} \mathrm{Hg}$ and oxygen saturation of $92 \%$. During the evacuation, the helicopter climbed to an altitude of 7000 feet (2134 meters), and the patient became more tachycardic, hypotensive, and his oxygen saturation dropped to $86 \%$ despite suctioning and subsequent transfer from the ventilator to manual ventilation. A rapid in-flight ultrasound examination revealed a pneumothorax. After needle decompression and chest tube placement the patient's vital signs normalized. This case report is the first of ultrasound being used to alter management of a patient during transport. As the author suggests, further studies would help define the utility of ultrasound in air evacuation.

(J Emer Medicine. 2009;1-4) JJ Madill.

Prepared by Anil Menon, MD, Stanford Wilderness Medicine

Fellow, Palo Alto, CA, USA

\section{JOURNAL OF APPLIED PHYSIOLOGY}

\section{Optic Nerve Sheath Diameter Correlates With The Presence and Severity of Acute Mountain Sickness: Evidence For Increased Intracranial Pressure}

It has been postulated that elevated intracranial pressure (ICP) causes acute mountain sickness (AMS). Elevated ICP might result from hypoxia induced cerebral vasodilation or edema. When attempting to correlate AMS with ICP, previous studies have not been able to adequately measure ICP or have been limited by sample size. This cross-sectional study sampled a larger population of climbers while using ultrasonagraphy to measure ICP.

Study participants were solicited from climbers at Pheriche, Nepal (4240 m) and 287 were enrolled. The participants did not climb higher than Pheriche for the 2 weeks preceding participation. One physician examined the patients and assessed AMS with the Lake Louise Score (LLS). A headache and LLS greater then 3 indicated AMS, and occurred in 69 patients (24\%).

A second, blinded physician performed the ultrasonagraphy. This physician used a high frequency probe $(7-10 \mathrm{MHz})$ to save 3 cross-sectional images of the optic nerve sheath from each eye in a supine patient whose eyes were closed. Sheath diameter was not calculated in real-time but images were saved and measured by a third observer at a later time. All 6 images were averaged at a point 3 millimeters posterior to the globe. In an effort to reduce interobserver differences, an additional blinded observer measured 40 randomly selected patients.

Optic nerve sheath diameter (ONSD) was correlated with the occurrence and severity of AMS (OR $6.32 \mathrm{P}<0.001)$ as assessed by the LLS. The authors suggest that ONSD might serve as a clinical and research tool for assessing AMS. A major drawback to this study was the lack of baseline low altitude ONSD measurements in the subjects.

(J Appl Physiol. 2009;106:1207-1211) PJ Fagenholz, JA Gutman, AF Murray, et al.

Prepared by Anil Menon, MD, Stanford Wilderness Medicine Fellow, Palo Alto, CA, USA 